\author{
Revista Arbitrada Interdisciplinaria KOINONIA \\ Año VI. Vol VI. N 12. Julio - Diciembre. 2021 \\ Hecho el depósito de Ley: FA2016000010 \\ ISSN: 2542-3088 \\ FUNDACIÓN KOINONIA (F.K). Santa Ana de Coro. Venezuela. \\ María Angélica Heredia-Quito; Cecilia Ivonne Narváez-Zurita \\ http://dx.doi.org/10.35381/r.k.v6i12.1288
}

\title{
La importancia de la evaluación del desempeño del talento humano en las organizaciones de salud
}

The importance of human talent performance evaluation in healthcare organizations

\author{
María Angélica Heredia-Quito \\ maria.heredia.18@est.ucacue.edu.ec \\ Universidad Católica de Cuenca, Cuenca \\ Ecuador \\ https://orcid.org/0000-0001-6471-7455 \\ Cecilia Ivonne Narváez-Zurita \\ inarvaez@ucacue.edu.ec \\ Universidad Católica de Cuenca, Cuenca \\ Ecuador \\ https://orcid.org/0000-0002-7437-9880
}

Recepción: 15 de marzo 2021

Revisado: 15 de mayo 2021

Aprobación: 15 de junio 2021

Publicación: 01 de julio 2021 


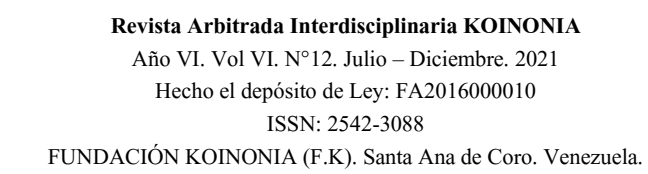

María Angélica Heredia-Quito; Cecilia Ivonne Narváez-Zurita

\title{
RESUMEN
}

El Capital humano es la columna vertebral del sistema de salud, su desarrollo implica procesos transversales y continuos de capacitación y actualización para fortalecer sus competencias en la gestión y atención integral. El objetivo es aplicar los procesos administrativos que la ley y las normativas del MDT faculta a las EODs, siendo necesario llevar a cabo una planificación actualizada del TTHH, seguida del levantamiento FAO de todos los funcionarios y por último solicitar a través del órgano regular la creación de partidas para la reubicación del personal. Se aplica una investigación descriptiva con diseño no experimental. Con los resultados obtenidos en la investigación realizada a los funcionarios del Hospital Homero Castanier Crespo, se ha propuesto aplicar el método $360^{\circ}$, de esta manera se podrá optimar el desempeño de los funcionarios del Hospital y mejorar la gestión administrativa.

Descriptores: Método de evaluación; formación profesional; planificación. (Palabras tomadas del Tesauro UNESCO).

\begin{abstract}
Human Capital is the backbone of the health system, its development implies transversal and continuous training and updating processes to strengthen its competencies in management and comprehensive care. The objective is to apply the administrative processes that the law and the regulations of the MDT empower the DOEs, being necessary to carry out an updated planning of the TTHH, followed by the FAO survey of all officials and finally request through the regular body the creation of items for the relocation of staff. A descriptive research with a non-experimental design is applied. With the results obtained in the investigation carried out on the officials of the Homero Castanier Crespo Hospital, it has been proposed to apply the $360^{\circ}$ method, in this way it will be possible to optimize the performance of the Hospital officials and improve administrative management.
\end{abstract}

Descriptors: Evaluation methods; vocational training; planning. (Words taken from the UNESCO Thesaurus). 
Revista Arbitrada Interdisciplinaria KOINONIA

Año VI. Vol VI. N 12. Julio - Diciembre. 2021

Hecho el depósito de Ley: FA2016000010

ISSN: $2542-3088$

FUNDACIÓN KOINONIA (F.K). Santa Ana de Coro. Venezuela.

María Angélica Heredia-Quito; Cecilia Ivonne Narváez-Zurita

\section{INTRODUCCIÓN}

El Ministerio de Salud Pública como la máxima Autoridad Sanitaria (AS), para cumplir con los objetivos del MAIS-FCI, organiza y construye los procesos con la finalidad de definir la ofertar las prestaciones de salud en los tres niveles de atención, cuya misión es la atención integral de las personas, familias y comunidades en un espacio poblacional determinado. Esta es la parte fundamental en la que se asienta la estructura del Sistema Nacional de Salud (SNGSP, 2012, p. 35).

En este sentido, Vance Mafla (2012) afirma. "como entidades dependientes del Ministerio de Salud Pública, los Hospitales establecen un modelo de gestión en red que permite satisfacer todas las necesidades de salud de forma integral, de calidad y gratuidad" ( $p$. 4). Por otro lado, el MSP como autoridad Sanitaria Nacional y con el objetivo de articular la Red Pública Integral de Salud y la Red Complementaria, homologa la tipología de los establecimientos de salud por niveles de atención del Sistema Nacional de Salud, que rige para todo el sector salud del país, clasificándose en primer nivel de atención, segundo nivel de atención, tercer nivel de atención, cuarto nivel de atención y atención especializada. (SNGSP, 2012, p. 85)

La planificación territorial que ha establecido el Ministerio de Salud Pública cuenta con 9 zonas, 140 distritos y 1134 circuitos. Las provincias de Azuay, Cañar y Morona Santiago pertenecen a la Zona de Salud 6, cuenta con tres hospitales generales: Vicente Corral Moscoso con 253 camas censables, Homero Castanier Crespo con 146 camas censables, y hospital de Macas con 70 camas censables. El Hospital Homero Castanier Crespo, es un hospital general de segundo nivel de complejidad dentro del Sistema Nacional de Salud con una capacidad instalada de 150 camas, en el año 2020 prestó atención a 88.225 usuarios, se encuentra ubicado en la ciudad de Azogues, capital de la Provincia del Cañar (Hospital Homero Castanier Crespo, 2021).

El elemento de gestión comprende los procesos gerenciales que brindan apoyo a la provisión y organización de los servicios de salud, para alcanzar beneficios en resultados 


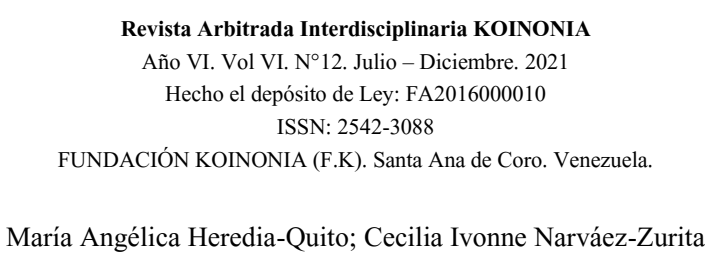

sanitarios dentro del argumento del Modelo de Atención Integral. Es así que el SNGSP (2012) afirma:

La gestión estratégica del talento humano es un aspecto fundamental para el Modelo Integral de Salud, se enmarca en la normativa nacional (LOSEP). El equipo de salud es la columna vertebral del sistema su desarrollo implica procesos transversales y continuos de capacitación y actualización para fortalecer sus competencias para la gestión y atención integral. (pág. 100)

Como Entidades que dependen del MSP, los hospitales crean un modelo de gestión en red las mismas permiten satisfacer de forma integral las necesidades de salud de manera gratuita y con calidad. La estructura que posee el MSP se basa en la filosofía y orientación de gestión por procesos, clientes, productos o servicios (Vance-Mafla, 2012). Al tratarse de una Unidad de 146 camas, el Hospital Homero Castanier Crespo es considerado un Hospital General de acuerdo con el "Estatuto Orgánico de Gestión Organizacional de Hospitales del Ministerio de Salud Pública" del Ecuador, en el que se señalan las Competencias, Facultades, Responsabilidades y Atribuciones de cada uno de los funcionarios contemplados en la estructura, cuenta con un total de 581 funcionarios divididos en 68 administrativos y 261 profesionales de la salud (Hospital Homero Castanier Crespo, 2021).

Al ser un Hospital de segundo nivel el Estatuto Orgánico de Hospitales del Ministerio de Salud Pública orden y clasifica en función al grado de contribución y cumplimiento de su misión y tenemos los procesos Gobernantes, procesos agregadores de valor y procesos Habilitantes de asesoría y de apoyo quienes apoyan la viabilización de la gestión Institucional (Vance-Mafla, 2012).

Actualmente el HHCC presenta un problema notable y es la falta de redistribución del personal en el área administrativa de acuerdo con el manual de puestos, en consecuencia existe una distribución incorrecta del personal en los diferentes departamentos, en este caso el objetivo de la Institución es aplicar los procesos administrativos que la ley y las normativas del MDT faculta a las EODs, siendo necesario llevar a cabo una planificación actualizada del TTHH, seguida del levantamiento FAO de todos los funcionarios y por 
Revista Arbitrada Interdisciplinaria KOINONIA

Año VI. Vol VI. N 12. Julio - Diciembre. 2021

Hecho el depósito de Ley: FA2016000010

ISSN: 2542-3088

FUNDACIÓN KOINONIA (F.K). Santa Ana de Coro. Venezuela.

María Angélica Heredia-Quito; Cecilia Ivonne Narváez-Zurita

último solicitar a través del órgano regular la creación de partidas para la reubicación del personal.

\section{Referencial Teórico}

\section{Desempeño laboral y el mejoramiento de las habilidades individuales}

Antes de centrarse en el tema materia de la presente investigación se debe considerar ciertos aspectos fundamentales que intervienen en la ejecución y cristalización de metas y objetivos de una organización, y uno de los más sustanciales es el trabajo y rendimiento del Talento Humano, siendo el desempeño laboral un punto de vital importancia (AmadorLicona, et al. 2018). En este sentido Recursos Humanos es el área, a la que también se la conoce como ingenio humano, interacciones industriales o capital humano, es la encargada de implantar mecanismos para elegir, formar, guiar al personal, y conseguir su mejor progreso, así también eleva su nivel de satisfacción y pertenencia en la organización. Poseer una organización con personal motivado es indispensable para obtener una mayor productividad, es así que las principales funciones del área del personal son:

1. Reclutamiento y selección

2. Administración de sueldos y salarios

3. Capacitación y desarrollo

4. Relaciones laborales

5. Higiene y seguridad industrial

6. Servicios y prestaciones

7. Planeación y evaluación (Münch, 2010).

El capital humano es mucho más que un componente beneficioso en los planes de la gestión, son miembros de sistemas sociales de muchas empresas, son clientes de bienes y servicios e influyen de forma esencial en la demanda, y son miembros de familias, escuelas, iglesias, asociaciones industriales y partidos políticos. En estas diversas funcionalidades, las personas establecen leyes que gobiernan a los administradores, 


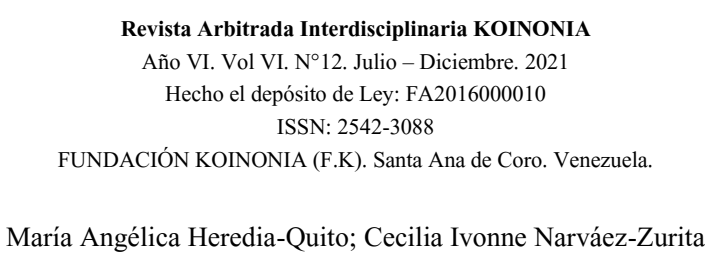

comportamientos éticos que guían una tradición de dignidad humana que es una característica fundamental de la sociedad de la cual formamos parte (Argudo-Tello, et al. 2019).

Si bien es cierto tanto las compañías como las empresas difieren en sus metas, puesto que es obvio que los empleados además poseen necesidades y fines que son especialmente relevantes para ellos; por medio de la capacidad de dirección, los directivos les ayudan a constatar que tienen la posibilidad de saciar sus propias necesidades y usar su habilidad a la vez que aportan con las metas de una organización. De esta forma, los administradores tienen que comprender las funcionalidades que asumen los individuos, y las individualidades y personalidades de la población (Koontz, et al. 2012). En cuanto al desempeño laboral, existen varias teorías al respecto teniendo entre los más destacados autores los siguientes:

Chiavenato (2000) afirma: "El desempeño es el comportamiento del evaluado en la búsqueda de los objetivos fijados. Constituye la estrategia individual para lograr los objetivos deseados" (pág. 126).

Por otra parte, Bittel (2000) plantea: Que el desempeño es influenciado en gran parte por las expectativas del empleado sobre el trabajo, sus actitudes hacia los logros y su deseo de armonía. Por tanto, el desempeño se relaciona o vincula con las habilidades y conocimientos. (p. 87)

Al respecto Pedraza, et al. (2010) sostienen: El desempeño laboral ha sido considerado como pieza fundamental para el éxito de una Institución; siendo así que hoy existe gran interés por parte de aquellos directivos de la administración del Talento Humano (p. 493) En ese marco el desempeño laboral son las conductas que se evidencian en cada uno de los funcionarios mismos que sobresalen para cumplir a cabalidad con la finalidad de cierta Institución y que permiten ser evaluados conforme el desarrollo de sus competencias y a su nivel de aportación a la Organización (Cordero, et al. 2019).

Una de las principales funciones del área de personal es el entrenamiento, capacitación y desarrollo, cuya finalidad es promover las habilidades, competencias y capacidades del 
Revista Arbitrada Interdisciplinaria KOINONIA

Año VI. Vol VI. N 12. Julio - Diciembre. 2021

Hecho el depósito de Ley: FA2016000010

ISSN: $2542-3088$

FUNDACIÓN KOINONIA (F.K). Santa Ana de Coro. Venezuela.

María Angélica Heredia-Quito; Cecilia Ivonne Narváez-Zurita

personal, y con ello obtener una mayor productividad, y desarrollen cada una de sus destrezas (Münch, 2010).

Es así que la educación profesional es la base para la preparación del ser humano en la vida profesional, la misma comprende 3 fases que se encuentran estrechamente relacionadas:

Formación profesional: es la educación profesional que recibe un individuo a fin de encontrarse preparado para el funcionamiento de un puesto, ya sea en áreas administrativas u operativas de una Organización.

Desarrollo profesional: perfecciona a la persona en sus destrezas y fomenta sus capacidades en una determinada carrera profesional, está dirigido para ejecutivos y directivos.

Capacitación: prepara al individuo para un cargo o funcionalidad a través de la captación de conocimientos y aptitudes, el presente está dirigido para niveles jerárquico e intermedios (Universidad de Champagnat, 2002).

Es común que en la mayoría de las organizaciones se considere a la capacitación como un mal primordial, debido a que a veces la norma laboral obliga a impartir capacitaciones anuales con un determinado número de horas-hombre, lamentablemente en las organizaciones no se planifica esta actividad, razón por la cual se carece de beneficios que podría aportar la misma. Es importante puntualizar que el entrenamiento, capacitación y el desarrollo tienen tal trascendencia siendo así que Ishikawa, considerado como precursor de la calidad total en Japón, manifestaba que la "calidad comienza con enseñanza y acaba con educación"; toda vez que la misma debería ser un proceso constante (Münch, 2010). Sin embargo, antes de considerar un programa o método de capacitación y desarrollo es necesario tomar en cuenta tres tipos de necesidades:

1. Necesidades de la Organización, como las metas y objetivos de la organización.

2. Necesidades afines con las operaciones que pueden determinarse a través de un manual de puestos y los modelos de desempeño. 


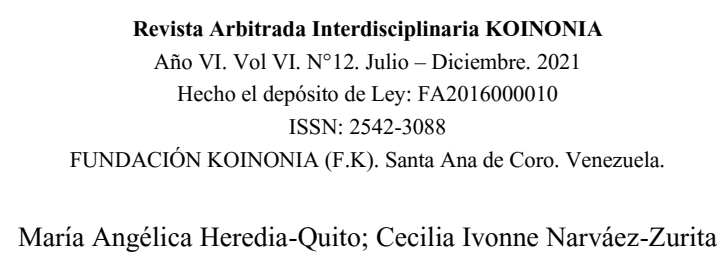

3. Necesidades de capacitación individuales que pueden manifestarse después de una evaluación de desempeño, entrevistas, encuestas y planes de carrera.

Es así que se debe estudiar con mayor detalle los pasos del proceso de desarrollo de los individuos (Koontz, et al. 2012). Por otra parte, el desarrollo involucra una enseñanza que va más allá de la labor diaria y tiene una dirección a largo plazo, dispone a sus empleados para perdurar al mismo ritmo de la empresa, mientras se modifica y crece (Wayne- Mondy \& Noe, 2005).

Es también relevante tomar en cuenta la motivación dentro del desarrollo y crecimiento de las personas y que actualmente es el centro de la reflexión teórico-práctico tanto de quienes investigan como de los administradores, el motivo es muy simple. La base del éxito de una organización es el conjunto o grupo de personas que la conforman; pero los trabajadores y empleados no se encuentran contentos ya sea con su trabajo o con los medios de promoción personal, social o con la misma empresa, por lo tanto, no se encuentran motivados. Así lo confirman varias investigaciones que en los últimos años han estudiado este problema (Sarries-Sanz \& Casares-García, 2008).

En conclusión, el Talento Humano indiscutiblemente tiene vital importancia en el normal desarrollo de las instituciones y es por eso que se debe encargar su dirección a personas que se encuentran debidamente capacitadas y calificadas a fin de lograr el cumplimiento del rol para el cual fue contratado, por ello concierne hablar de cómo un funcionario se comporta y ejecuta su labor y aquello cómo impacta en la organización, tanto positivamente como negativamente. Justamente es por esa razón que resulta sumamente importante medirlo y evaluarlo. En efecto, un mal manejo puede influir en la organización, pero uno bueno es capaz de incrementar la motivación y acercarnos a nuestro objetivo final. 
Revista Arbitrada Interdisciplinaria KOINONIA

Año VI. Vol VI. N 12. Julio - Diciembre. 2021

Hecho el depósito de Ley: FA2016000010

ISSN: $2542-3088$

FUNDACIÓN KOINONIA (F.K). Santa Ana de Coro. Venezuela.

María Angélica Heredia-Quito; Cecilia Ivonne Narváez-Zurita

\section{Procedimientos de medición y evaluación del desempeño laboral en la organización.}

En este sentido, se tiene que el artículo 76 de la LOSEP (2020) prescribe:

Que el subsistema de evaluación del desempeño es el conjunto de normas, técnicas, métodos, protocolos y procedimientos armonizados, justos, transparentes, imparciales y libres de arbitrariedad que sistémicamente se orienten a evaluar bajo parámetros objetivos acordes con las funciones, responsabilidades y perfiles del puesto; y, que la evaluación se fundamentará en indicadores cuantitativos y cualitativos de gestión, encaminados a impulsar la consecución de los fines y propósitos institucionales, el desarrollo de los servidores públicos y el mejoramiento continuo de la calidad del servicio público, prestado por todas las instituciones señaladas en el artículo 3 de esta Ley (p. 64).

A título ilustrativo es pertinente conocer el origen, evolución y alcance de la evaluación del desempeño como práctica de administración humana en los centros de trabajo se remonta al siglo XIX, se ubica como un pionero a Robert Owen (1771-1858), considerado en su etapa un hito en la revolución industrial, el mismo realizó ensayos en la fábrica de New Lannark, en los cuales plasmó sus ideas sobre el valor de la motivación y el clima gremial en la organización, Charles Babbage (1792-1871), en su creación "Economía de las Máquinas y Manufacturas", explica que es necesario examinar los métodos y precios de producción y usar técnicas de estudios del tiempo de ejecución para minimizar la era de aprender el trabajo y el desperdicio de material en la fase de aprendizaje (Cejas, et al. 2012).

Posteriormente en 1842 el gobierno de los EEUU por medio del Congreso establece una ley en la se debía evaluar anualmente a sus trabajadores de manera obligatoria en todas las empresas, de esta manera en el siglo XX los métodos de evaluación aplicadas eran cada vez más avanzadas (Alfonso, 2016). Es necesario conceptualizar las propiedades o componentes sobre los cuales se va a evaluar el manejo de una persona. Estas propiedades o componentes de evaluación tienen que consumar con reglas minúsculas que garanticen la validez del sistema, las cuales se dicen luego: 
Revista Arbitrada Interdisciplinaria KOINONIA

Año VI. Vol VI. N 12. Julio - Diciembre. 2021

Hecho el depósito de Ley: FA2016000010

ISSN: $2542-3088$

FUNDACIÓN KOINONIA (F.K). Santa Ana de Coro. Venezuela.

María Angélica Heredia-Quito; Cecilia Ivonne Narváez-Zurita

- Las propiedades o componentes de evaluación tienen que ser importantes representativas para realizar de manera correcta las funcionalidades del puesto que domina el evaluado.

- Tienen que ser específicos para las funcionalidades de todos los cargos considerados en la calificación.

- Deben ser tan equitativos y medible.

- Reducir la cantidad excesiva de características, ya que obstaculiza y hace más difícil aplicar el sistema de evaluación (Brazzolotto, 2012).

En cuanto a la responsabilidad de la evaluación de desempeño se asigna a varias delegaciones, conforme con la política realizada en temas de $\mathrm{RRHH}$. En algunas ocasiones se atribuye a un organismo Staff correspondiente al área de $\mathrm{RRHH}$, en otras ocasiones se asigna a una comisión de evaluación (Carrión-Agila, et al. 2019). En efecto el proceso de llevar a cabo un sistema de evaluación del desempeño es importantísimo por lo que antes de aplicarla se debe considerar los siguientes recursos y pasos:

- Examinar cuáles son los indicadores que se evaluaran

- Optar por un método a través de parámetros

- Crear la encuesta para acelerar la gestión

- Entregar la evaluación: entregar la encuesta a los empleados y promotores de esta manera asegurar que se rellena en un ámbito conveniente, independiente de sesgos.

- Hacer el seguimiento, para verificar que se está ejecutando.

- Medir los resultados y crear informes.

- Comunicar los resultados de forma asertiva y con absoluta transparencia 
Revista Arbitrada Interdisciplinaria KOINONIA

Año VI. Vol VI. N 12. Julio - Diciembre. 2021

Hecho el depósito de Ley: FA2016000010

ISSN: 2542-3088

FUNDACIÓN KOINONIA (F.K). Santa Ana de Coro. Venezuela.

María Angélica Heredia-Quito; Cecilia Ivonne Narváez-Zurita

Finalmente, los métodos de evaluación que miden al personal se clasifican de acuerdo con sus características, conducta y resultados.

1. El método basado en características está creado para medir hasta qué punto un funcionario tiene ciertas cualidades como fiabilidad, creatividad, nuevas ideas o liderazgo, para el presente y futuro de dicha organización

2. Método de escalas mixtas: Es una transformación del procedimiento de escala básica. En vez de evaluar las cualidades con una escala se provee al evaluador de tres descripciones concretas de cada cualidad: superior, promedio e inferior.

3. Método de distribución forzada: Pide que el evaluador elija entre varias declaraciones, constantemente puestas a modo de pares que parecen por igual favorables y desfavorables.

4. Método de formas narrativas: Es necesario que el evaluador prepare un estudio que detalle que realiza evaluaciones muy precisas.

5. Método del comportamiento: comportamiento, la misma permite al evaluador identificar de manera inmediata el momento que un empleado se separa de la escala, sirve para describir de forma concreta las acciones que se deberían (o no deberían) exponer en el puesto.

6. Y por último el procedimiento basado en resultados se centra en evaluar los logros obtenidos por los empleados en el lugar de trabajo. Sus defensores aseguran que son más ecuánimes que otros procedimientos y conceden mayor atributo a los empleados.

Como se señaló anteriormente la evaluación del desempeño no es un fin en sí, sino un instrumento, una herramienta para mejorar los resultados de los recursos humanos de la institución, que vez coordinado, planificado y desarrollado el proyecto de evaluación de desempeño se obtiene beneficios a corto mediano y largo plazo. Los beneficiarios directos son el individuo, el jefe, la empresa y la sociedad (Buelvas, et al. 2002). 
Revista Arbitrada Interdisciplinaria KOINONIA

Año VI. Vol VI. N 12. Julio - Diciembre. 2021

Hecho el depósito de Ley: FA2016000010

ISSN: $2542-3088$

FUNDACIÓN KOINONIA (F.K). Santa Ana de Coro. Venezuela.

María Angélica Heredia-Quito; Cecilia Ivonne Narváez-Zurita

\section{Evaluación de desempeño, orientados a lograr metas y objetivos de la organización.}

Antes de adentrarnos en el tema de evaluación hablaremos sobre el propósito de las organizaciones como facilitar las labores, organizar y perfeccionar las actividades y recursos para que los procesos sean fluidos y faciliten el trabajo para aquello que laboran en la misma, de esta manera obtener la satisfacción de los usuarios internos y externos. Todas las organizaciones están destinadas a conseguir metas y objetivos los mismos deben ser medibles y alcanzables a corto, mediano y largo plazo de tiempo (Münch, 2010).

Históricamente la evaluación del desempeño ha impactado a la administración del recurso humano, se alinea al marco filosófico de la organización, formado por la tarea, perspectiva, fines, y valores, para garantizar la pertinencia en la aplicación de la evaluación del manejo en empresas públicas o privadas, la formulación de tácticas organizacionales se apalanca en valores organizacionales que tienen que caracterizar al ingenio humano de la organización, de forma que no es viable conseguir resultados sino es por medio de la población, ahí el costo añadido del seguimiento y evaluación del funcionamiento personal. Aprender y examinar por qué evaluar el rendimiento del trabajador, en qué magnitudes y bajo qué criterios delimita su alcance y orienta la finalidad de la evaluación del desempeño (Urgilés-Urgilés, et al. 2019). Los posibles objetivos que se alcanzan con un buen sistema de evaluación formal son:

1. Desarrollo: Se conoce que los trabajadores necesitan capacitarse más y ayuda a evaluar los resultados de los programas de capacitación.

2. Motivación: Promueve la iniciativa, despierta un sentimiento de responsabilidad y estimula los esfuerzos de desempeñarse mejor.

3. Comunicaciones: Base principal para mantener una buena comunicación entre jefe y subordinado en temas relacionados con el trabajo.

4. Respeto de la ley: Sirve como defensa legal de los ascensos, transferencias recompensas y despidos. 


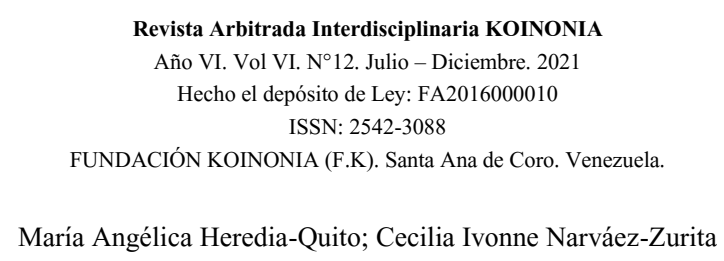

5. Investigación de ARH: Útil para validar las herramientas de selección, como los exámenes (Ivancevich, 2004).

La medición del desempeño sirve principalmente para:

1. Promover valores de igualdad y justicia, en los sistemas salariales que motiven la constancia y compromiso de los funcionarios en la organización.

2. Crear programas de educación y desarrollo que permita al trabajador mejorar su desempeño y desarrollo Identificando a potenciales funcionarios para promociones, traspasos o sucesión con nuevas responsabilidades.

3. Validar la eficiencia de los métodos y prácticas elaboradas en los cargos de la organización y promover la optimización permanente.

4. Los funcionarios que conforman la organización deben ser reconocidos por sus principios y valores Organizacionales.

5. Establecer estrategias y metas tomando decisiones que faciliten la gestión del Talento Humano.

6. Jefes y trabajadores deben mantener un sistema de comunicación y retroalimentación fortalecida (Cejas, et al. 2012).

Así también la evaluación de desempeño no trabaja sola, siempre se encuentra interrelacionada con otros subsistemas de recursos humanos como son:

El análisis y descripción de puestos, remuneraciones y beneficios, desarrollo y planes de sucesión y la formación (Alles, 2006). Un sistema de evaluación del desempeño orienta la administración de recursos humanos en forma incorporada y alineada a las tácticas organizacionales, los resultados representan insumos que retroalimentan y enriquecen a otros sistemas como: selección, educación, desarrollo, indemnización y beneficios e interrelaciones laborales, entender la conducta personal es, por consiguiente, necesaria para una gestión eficaz. 
Existe factores de éxito como se demuestra en la figura 1 al aplicar la Evaluación de desempeño, por lo tanto, recursos humanos debe asegurar lo siguiente:

- A partir de un entorno organizacional, la filosofía de las empresas, sea esta pública o privada, compañía u organización del gobierno, la cual debería tener una clara definición de la tarea y perspectiva, de esta forma como de fines organizacionales y eje estratégico para detectar lo cual se espera de la población en el funcionamiento de sus funcionalidades.

- Garantizar que las magnitudes a evaluar sean claras, medibles y observables a lo largo del funcionamiento de las funcionalidades de cada cargo de la composición organizacional.

- Implicar a toda la organización en las prácticas de evaluar el desempeño asegurando la ayuda de los diferentes actores a partir de la gerencia hasta el ocupante del último cargo de la composición organizacional.

En la figura 1 se puede observar los factores de éxito de una evaluación de desempeño.

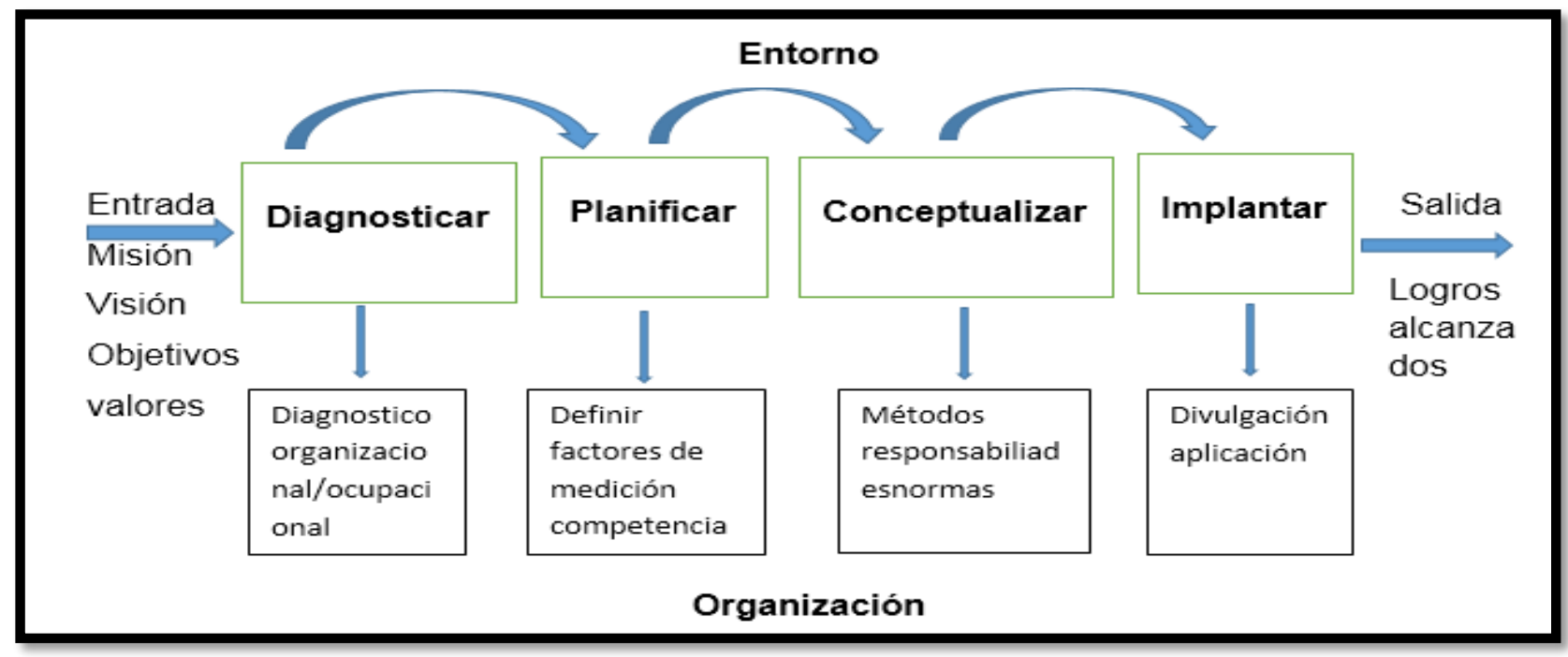

Figura 1. Factores de éxito.

Fuente: Saldoval (2010). 
Revista Arbitrada Interdisciplinaria KOINONIA

Año VI. Vol VI. N 12. Julio - Diciembre. 2021

Hecho el depósito de Ley: FA2016000010

ISSN: 2542-3088

FUNDACIÓN KOINONIA (F.K). Santa Ana de Coro. Venezuela.

María Angélica Heredia-Quito; Cecilia Ivonne Narváez-Zurita

La administración de RRHH es parte de un procedimiento, que es la misma empresa, y trabaja de manera abierta impulsando a otros procesos involucrados, a la vez retroalimentándose del ámbito empresarial. Esta información garantiza que cada integrante de la empresa conozca técnicamente sus responsabilidades y las competencias que debería enseñar a lo largo del desempeño de sus actividades, en medio de las que se resaltan:

Establecer etapas de medición formales como máximo dos veces al año, y entablar evaluaciones informales habituales, sin medir, como una posibilidad de comprobar los adelantos y/o reorientar actividades.

La gerencia debe contemplar que para una evaluación de desempeño eficiente existe dos objetivos: primero promover la meta de la evaluación de desempeño, con reuniones, talleres haciendo hincapié en las ventajas que tuvieran los empleados, supervisores o líderes y las unidades de $\mathrm{RRHH}$; el segundo, describir el procedimiento y la técnica de aplicación de la evaluación, triunfando la satisfacción y la ayuda de los individuos de la empresa.

Desde el punto de vista de la gerencia, ¿hacia dónde vamos?, ¿qué hacemos? y ¿cómo lo hacemos? las repuestas a estas cuestiones permiten conocer los componentes de triunfo que orientan e impulsan a la organización (Cejas, et al. 2012).

\section{MÉTODO}

Con el fin de llevar a cabo el objetivo de estudio sobre la aplicación de la evaluación de desempeño al personal del Hospital Homero Castanier Crespo y obtener resultados óptimos, se realizó una investigación descriptiva con diseño no experimental. A fin de recopilar información se aplicó la técnica de las encuestas creando un cuestionario que permitan obtener respuestas claras para analizarlas estadísticamente, serán realizadas al personal Administrativo del Hospital las mismas permitirán conocer la experiencia de los servidores públicos en sus puestos de trabajo. 
Revista Arbitrada Interdisciplinaria KOINONIA

Año VI. Vol VI. N 12 . Julio - Diciembre. 2021

Hecho el depósito de Ley: FA2016000010

ISSN: 2542-3088

FUNDACIÓN KOINONIA (F.K). Santa Ana de Coro. Venezuela.

María Angélica Heredia-Quito; Cecilia Ivonne Narváez-Zurita

El universo de estudio para esta investigación son los servidores públicos del Hospital Homero Castanier Crespo que se encuentran laborando en las diferentes áreas administrativas de esta Casa de Salud, conformando un universo de 60 funcionarios, divididos en la Dirección Administrativa Financiera, Compras Públicas, Asesoría Jurídica, Talento Humano, Activos Fijos, Mantenimiento, Planificación, Trabajo Social, Dirección Médica y la Unidad de TICS.

El proceso de obtención de datos se gestionó por medio de un cuestionario elaborado en la plataforma de formularios de Google (https://www.google.com/forms/); se establecieron preguntas cerradas y politómicas y recolectando las respuestas de los encuestados en porcentajes; para los gráficos se utilizó el sistema Excel versión 2019, los que evidencian la construcción de la información, analizándose los datos mediante la estadística descriptiva (Bernal-Avila, et al. 2019).

\section{RESULTADOS}

A continuación, se muestra la Tabla 1 y la figura 2 con el resumen de los resultados conseguidos, clasificados según las variables determinadas en el instrumento de investigación aplicado: 
Revista Arbitrada Interdisciplinaria KOINONIA

Año VI. Vol VI. N 12. Julio - Diciembre. 2021

Hecho el depósito de Ley: FA2016000010

ISSN: 2542-3088

FUNDACIÓN KOINONIA (F.K). Santa Ana de Coro. Venezuela.

María Angélica Heredia-Quito; Cecilia Ivonne Narváez-Zurita

Tabla 1.

Resultados de la encuesta aplicada al personal administrativo del Hospital Homero Castanier Crespo.

\begin{tabular}{|c|c|c|c|}
\hline & & \multicolumn{2}{|c|}{ Resultado \% } \\
\hline Variable & Pregunta & $\mathbf{S i}$ & No \\
\hline $\begin{array}{l}\text { Formación } \\
\text { académica }\end{array}$ & $\begin{array}{l}\text { ¿Considera que } \\
\text { su formación } \\
\text { académica, } \\
\text { está acorde con } \\
\text { las funciones } \\
\text { que } \\
\text { desempeña? }\end{array}$ & $68,3 \%$ & $31,7 \%$ \\
\hline $\begin{array}{l}\text { Actividades } \\
\text { laborales }\end{array}$ & $\begin{array}{l}\text { ¿Las } \\
\text { actividades que } \\
\text { realiza tiene } \\
\text { relación con su } \\
\text { contrato de } \\
\text { trabajo? }\end{array}$ & $45 \%$ & $55 \%$ \\
\hline $\begin{array}{l}\text { Tiempo de la } \\
\text { jornada } \\
\text { laboral }\end{array}$ & $\begin{array}{l}\text { ¿Se encuentra } \\
\text { ocupado en sus } \\
\text { tareas durante } \\
\text { la mayor parte } \\
\text { de la jornada } \\
\text { laboral? }\end{array}$ & $51,7 \%$ & $48,3 \%$ \\
\hline $\begin{array}{l}\text { Retribución } \\
\text { económica }\end{array}$ & $\begin{array}{l}\text { ¿La retribución } \\
\text { económica que } \\
\text { recibe, está } \\
\text { acorde a las } \\
\text { funciones que } \\
\text { realiza? }\end{array}$ & $76,7 \%$ & $23,3 \%$ \\
\hline
\end{tabular}

Fuente: Investigación de campo 
Revista Arbitrada Interdisciplinaria KOINONIA

Año VI. Vol VI. N 12. Julio - Diciembre. 2021

Hecho el depósito de Ley: FA2016000010

ISSN: 2542-3088

FUNDACIÓN KOINONIA (F.K). Santa Ana de Coro. Venezuela.

María Angélica Heredia-Quito; Cecilia Ivonne Narváez-Zurita

En la figura 2 se puede observar el resultado obtenido según el número de encuestados

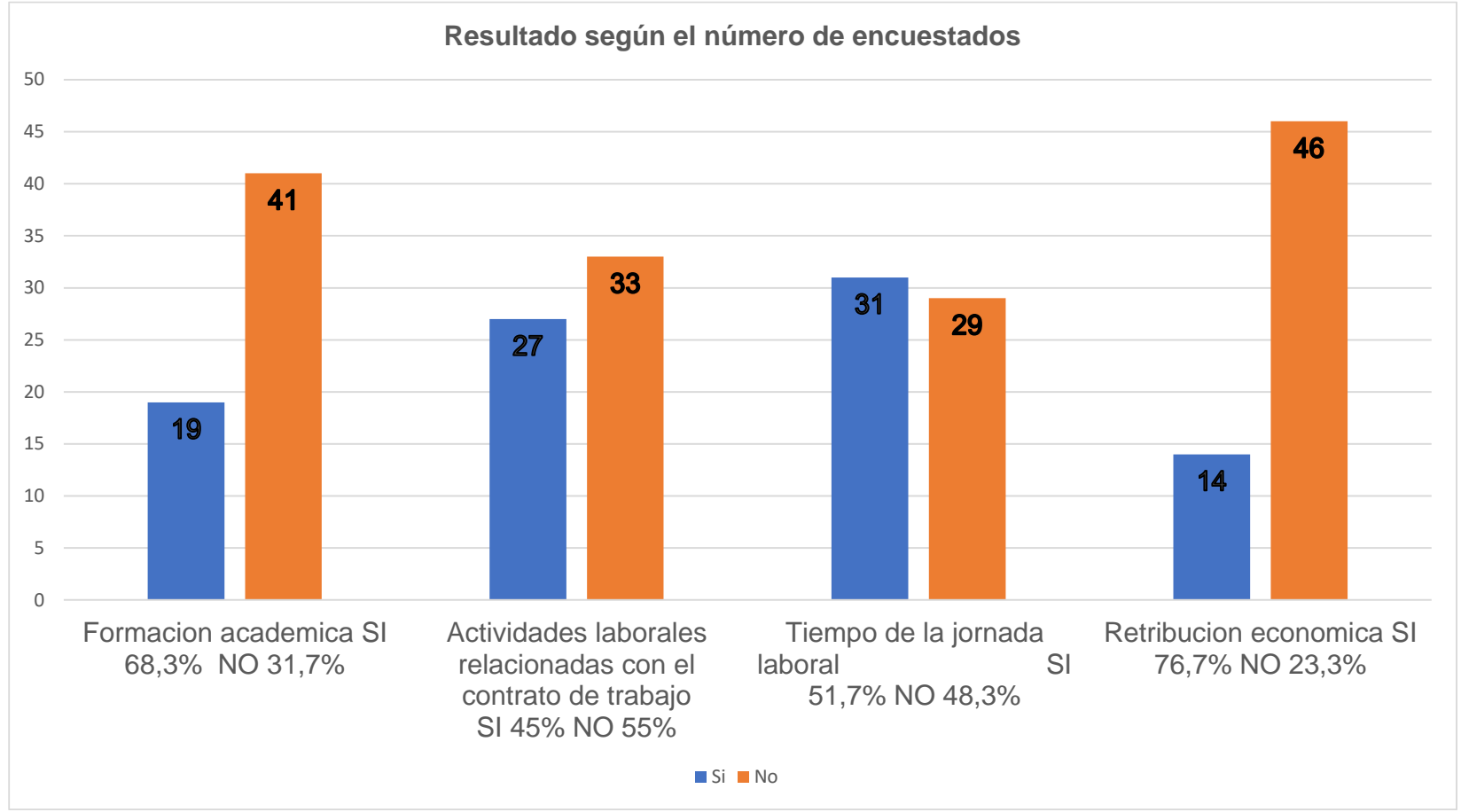

Figura 2: Resultado según el número de encuestados.

Fuente: Investigación de campo.

Al obtener los resultados de la investigación realizada en el Hospital Homero Castanier Crespo se puede evidenciar lo siguiente:

1. Respecto a la formación académica se observa que de manera mayoritaria los funcionarios no cumplen con la formación académica para los puestos de trabajo que en la actualidad desempeñan, ya sea porque sus perfiles profesionales son superior o inferior al perfil del puesto.

2. A si también se evidencia que las actividades laborales que realizan en su mayoría no se encuentran acorde a su contrato de trabajo, toda vez que varios de ellos poseen un cambio administrativo por una supuesta necesidad Institucional, así 
Revista Arbitrada Interdisciplinaria KOINONIA

Año VI. Vol VI. N 12. Julio - Diciembre. 2021

Hecho el depósito de Ley: FA2016000010

ISSN: 2542-3088

FUNDACIÓN KOINONIA (F.K). Santa Ana de Coro. Venezuela.

María Angélica Heredia-Quito; Cecilia Ivonne Narváez-Zurita

como también no existe las partidas presupuestarias correspondientes o las mismas se encuentran ocupadas por funcionarios que desarrollan una actividad diferente.

3. Un tema muy importante que se denota es el descontento marcado al dar a conocer que la retribución económica que perciben no está acorde al trabajo que realizan por varios factores como son la falta de reclasificación de las partidas presupuestarias, deficiencia por falta de gestión de partidas indispensables para cambios de partidas a los funcionarios que les pertenece y por la mala administración, la omisión o desconocimiento de los funcionarios de la Unidad de Talento Humano del Manual de descripción y valoración y clasificación de puestos de Hospitales y Establecimientos de Salud.

La presente investigación puede ser corroborada por la Contraloria General del Estado (2020) en la misma manifiesta que los Gerentes del Hospital Homero Castanier Crespo, que actuaron en los periodos comprendidos entre el 1 de febrero de 2016 y el 5 de enero de 2018; legalizaron acciones de personal, de servidores con nombramiento provisional, sin verificar y exigir al Responsable de Talento Humano, un informe técnico que justifique la designación del cargo y la remuneración a recibir, conforme lo establece el Manual de Descripción, valoración y clasificación de puestos de Hospitales, ocasionando que se eroguen valores por concepto de remuneraciones mensuales superior a lo establecido en el citado manual.

Con lo antes expuesto se confirma que en la Institución no se aplica de manera correcta el Manual de Descripción, valoración y clasificación de puestos de Hospitales, y que en la actualidad ya ha repercutido de manera personal, laboral y económica a varios funcionarios de esta Casa de Salud. 


\section{PROPUESTA}

Después de los resultados obtenidos en la investigación realizada a los funcionarios del Hospital Homero Castanier Crespo, se ha planteado aplicar el método $360^{\circ}$, debido a que es un método sencillo, práctico, eficiente, técnico y manual; para medir, examinar y desarrollar las capacidades, sapiencias y conductas de los servidores públicos del Nosocomio lo cual dejará mejorar el modelo de gestión administrativa y tomar decisiones con informes claros, pertinentes y honestos, en función a las competencias y perfiles del personal, como se puede observar en la figura 3, la misma involucra la autoevaluación, la evaluación interna y externa, a fin de destacar los limitantes reconocidos, y alcanzar una reorganización del personal administrativo acorde al Manual de Descripción, valoración y clasificación de puestos de Hospitales.

En la figura 3 se observa el proceso de Evaluación de desempeño a utilizar en el Hospital Homero Castanier Crespo

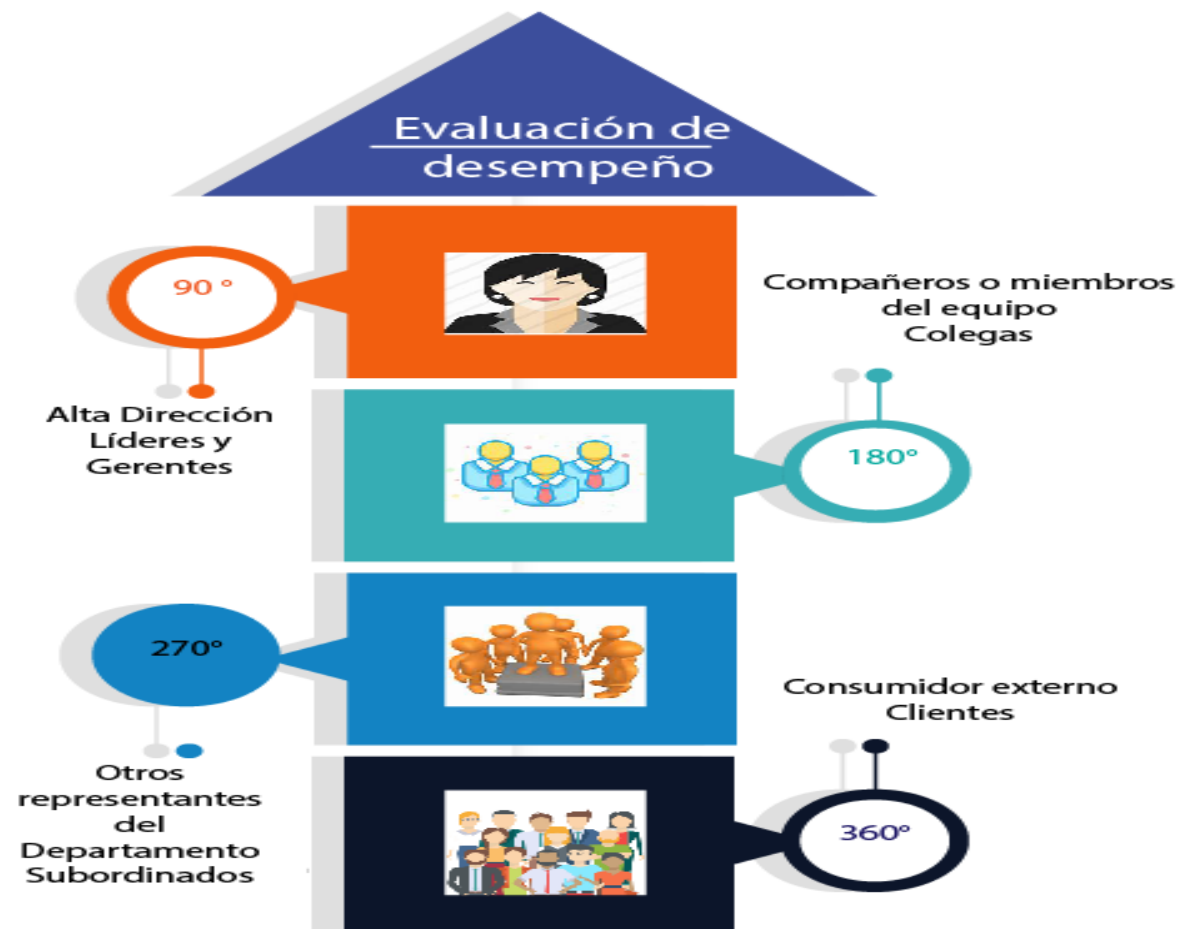

Figura 3. Esquema de propuesta de evaluación. Elaboración: Los autores. 
Revista Arbitrada Interdisciplinaria KOINONIA

Año VI. Vol VI. N 12. Julio - Diciembre. 2021

Hecho el depósito de Ley: FA2016000010

ISSN: 2542-3088

FUNDACIÓN KOINONIA (F.K). Santa Ana de Coro. Venezuela.

María Angélica Heredia-Quito; Cecilia Ivonne Narváez-Zurita

\section{Alta Dirección Líderes y Gerentes}

\section{El Hospital Homero Castanier Crespo se encuentra formado por el Gerente como máxima} autoridad, es quien deberá tomar la iniciativa y coordinar con su equipo de trabajo la socialización de las funciones y competencias.

A continuación, se puede observar en la figura 4, las variables indispensables para la elaboración de la propuesta:

\begin{tabular}{|c|c|c|}
\hline \\
\hline & \multirow{2}{*}{\multicolumn{2}{|c|}{ Jefe Inmediato: }} \\
\hline \multicolumn{2}{|r|}{ Remplazo en caso de ausencia: } & \\
\hline \multicolumn{3}{|c|}{ Le reemplaza a: } \\
\hline \multicolumn{3}{|c|}{ Responsabilidades } \\
\hline 1 & Elaborar rol de pagos de las fábricas & $\begin{array}{l}2 \text { Elaborar plantillas de prestamos quirografarios, fondos } \\
\text { de reserva y presentar al área financiera para el pago }\end{array}$ \\
\hline 3 & Reportar el pago a proveedores & \begin{tabular}{l|l} 
& Mantener informado al jefe de TTHH, sobre cualquier \\
aspecto relacionado con el personal
\end{tabular} \\
\hline & $\begin{array}{l}\text { Controlar y pagar vacaciones de las } \\
\text { fábricas }\end{array}$ & \begin{tabular}{l|l} 
Control quincenal de horas laboradas de los \\
trabajadores
\end{tabular} \\
\hline 7 & $\begin{array}{l}\text { Recibir, ingresar y verificar las facturas } \\
\text { de los proveedores }\end{array}$ & \begin{tabular}{l|l} 
& Realizar las actividades definidas en los procedimientos \\
$\mathbf{8}$ & del sistema de gestión de calidad
\end{tabular} \\
\hline \multicolumn{3}{|r|}{ Competencias } \\
\hline & Nivel de Competencia & \begin{tabular}{|c|} 
Definición de la Competencia \\
\end{tabular} \\
\hline & Inicial & \multirow{3}{*}{$\begin{array}{l}\text { Esta es la competencia que significa rápida ejecutividad } \\
\text { ante las pequeñas dificultades o problemas que surgen en } \\
\text { el día a día de la actividad. Supone actuar proactivamente } \\
\text { cuando ocurren desviaciones o dificultades sin esperar } \\
\text { efectuar tods las consultas en la líne jerárquica, evitando así } \\
\text { el agravamiento de problemas de importancia menor. } \\
\text { Implica tambien la capacidad de proponer mejoras sin que } \\
\text { haya un problema concreto que deba ser solucionado. }\end{array}$} \\
\hline & Competencia & \\
\hline & Iniciativa- Autonomía & \\
\hline & Grado de Competencia & Descripción de la categoría de la Competencia \\
\hline \multicolumn{2}{|r|}{ B } & \multirow{5}{*}{$\begin{array}{l}\text { Actúa para resolver los pequeños problemas diarios. En } \\
\text { ocaciones propone mejoras aunque no haya un problema } \\
\text { concreto que necesite solución. }\end{array}$} \\
\hline & Definición del grado de Competencia & \\
\hline \begin{tabular}{|l|l}
$\mathbf{A}$ \\
$\mathbf{B}$
\end{tabular} & \begin{tabular}{|l} 
Alto \\
Bueno, por encima del estandar
\end{tabular} & \\
\hline & $\begin{array}{l}\text { Mínimo necesario para el puesto( dentro } \\
\text { del perfil requerido) }\end{array}$ & \\
\hline \multirow{2}{*}{\multicolumn{3}{|c|}{\begin{tabular}{c|c} 
& Grado mínimo o insatisfactorio \\
Nivel de Competencia
\end{tabular}}} \\
\hline & & \\
\hline \multicolumn{2}{|r|}{ Inicial } & \multirow{3}{*}{$\begin{array}{l}\text { Esta competencia está asociada al compromiso con que las } \\
\text { personas realizan las tareas encomendadas. }\end{array}$} \\
\hline & Competencia & \\
\hline \multicolumn{2}{|r|}{ Responsabilidad } & \\
\hline & Nivel de Competencia & Definición de la Competencia \\
\hline & Inicial & \multirow{4}{*}{$\begin{array}{l}\text { Es la tendencia a loros de resultados, fijando metas } \\
\text { desafiantes por encima de estos estándares, mejorando y } \\
\text { manteniendo altos niveles de rendimiento en el marco de } \\
\text { las estrategias de la organización } \\
\text { Definición de la Competencia }\end{array}$} \\
\hline & Competencia & \\
\hline & Orientación a los resultados & \\
\hline & Nivel de Competencia & \\
\hline & $\frac{\text { Inicial }}{\text { Competencia }}$ & \multirow{2}{*}{$\begin{array}{l}\text { Es la capacidad de trabajar en colaboración con grupos } \\
\text { multidiciplinarios, con otras áreas de la organización u } \\
\text { organismoa externos con los que debe interactuar. }\end{array}$} \\
\hline \multicolumn{2}{|r|}{ Capacidad de planificación y organización } & \\
\hline & Nivel de Competencia & \begin{tabular}{|c|} 
Definición de la Competencia \\
\end{tabular} \\
\hline & $\begin{array}{c}\text { Gerencial } \\
\text { Competencia }\end{array}$ & \multirow{2}{*}{$\begin{array}{l}\text { Capacidad para analizar diversas variantes u opciones, } \\
\text { considerar las circunstancias existentes, los recursos } \\
\text { disponibles y su impacto en el negocio, para luego } \\
\text { seleccionar la alternativa más adecuada, con el fin de lograr } \\
\text { el mejor resultado en función de los objetivos } \\
\text { Institucionales. }\end{array}$} \\
\hline & Toma de decisión & \\
\hline
\end{tabular}

Figura 4. Matriz de Identificación del cargo y competencias.

Fuente: A partir de los autores (Ortiz-Andrade \& Rivas-Quezada, 2011).

Elaboración: Los autores. 
Revista Arbitrada Interdisciplinaria KOINONIA

Año VI. Vol VI. Nº12. Julio - Diciembre. 2021

Hecho el depósito de Ley: FA2016000010

ISSN: 2542-3088

FUNDACIÓN KOINONIA (F.K). Santa Ana de Coro. Venezuela.

María Angélica Heredia-Quito; Cecilia Ivonne Narváez-Zurita

Es importante que el Gerente del Hospital participe de manera activa en la planificación de la evaluación en conjunto con representantes de los funcionarios a ser evaluados y evaluadores, con el objetivo de tomar decisiones consensuadas para obtener una ejecución favorable de la evaluación.

\section{Tabla 2.}

Pasos a seguir del proceso de Planificación participativa.

\begin{tabular}{|c|c|}
\hline \multicolumn{2}{|c|}{ Proceso de Planificación participative } \\
\hline $\begin{array}{l}\text { Preparación y } \\
\text { organización }\end{array}$ & $\begin{array}{l}\text { Identificar las actividades a realizar para el } \\
\text { proceso de evaluación }\end{array}$ \\
\hline $\begin{array}{l}\text { Diagnóstico de la } \\
\text { situación }\end{array}$ & Análisis FODA del Hospital \\
\hline Plan de Desarrollo & $\begin{array}{l}\text { Identificar la secuencia de las actividades a } \\
\text { realizar principales y secundarias }\end{array}$ \\
\hline \multirow{4}{*}{$\begin{array}{l}\text { Plan de ejecución y } \\
\text { administración }\end{array}$} & 1. Actividades \\
\hline & 2. Responsible \\
\hline & 3. Tiempo \\
\hline & 4.Recursos \\
\hline Seguimiento y control & $\begin{array}{l}\text { 1. Responsable de realizar el monitoreo y } \\
\text { control }\end{array}$ \\
\hline & 2. Existencia de recursos \\
\hline
\end{tabular}

Fuente: A partir de los autores Coronel-Salamea, Bermeo-Pazmiño, \& Narváez-Zurita (2020).

Elaboración: Los autores. 
Revista Arbitrada Interdisciplinaria KOINONIA

Año VI. Vol VI. N 12. Julio - Diciembre. 2021

Hecho el depósito de Ley: FA2016000010

ISSN: 2542-3088

FUNDACIÓN KOINONIA (F.K). Santa Ana de Coro. Venezuela.

María Angélica Heredia-Quito; Cecilia Ivonne Narváez-Zurita

\section{Compañeros o miembros del equipo}

En los método de evaluación de $180^{\circ}$ y $270^{\circ}$ participan colegas de trabajo, subordinados y gerente, forman parte del proceso interno de la ejecución de la propuesta de evaluación de desempeño partiendo del establecimiento de los objetivos estratégicos, la planificación de un cronograma, capacitación a los evaluadores, una vez que se haya conseguido la información se continua con el respectivo análisis y se realiza la retroalimentación de los resultados obtenidos a los funcionarios que fueron evaluados, seguido de la preparación de la propuesta del Plan de mejora.

En el siguiente flujograma se puede observar la secuencia para llevar a cabo la ejecución de la evaluación. 
Revista Arbitrada Interdisciplinaria KOINONIA

Año VI. Vol VI. N 12 . Julio - Diciembre. 2021

Hecho el depósito de Ley: FA2016000010

ISSN: 2542-3088

FUNDACIÓN KOINONIA (F.K). Santa Ana de Coro. Venezuela.

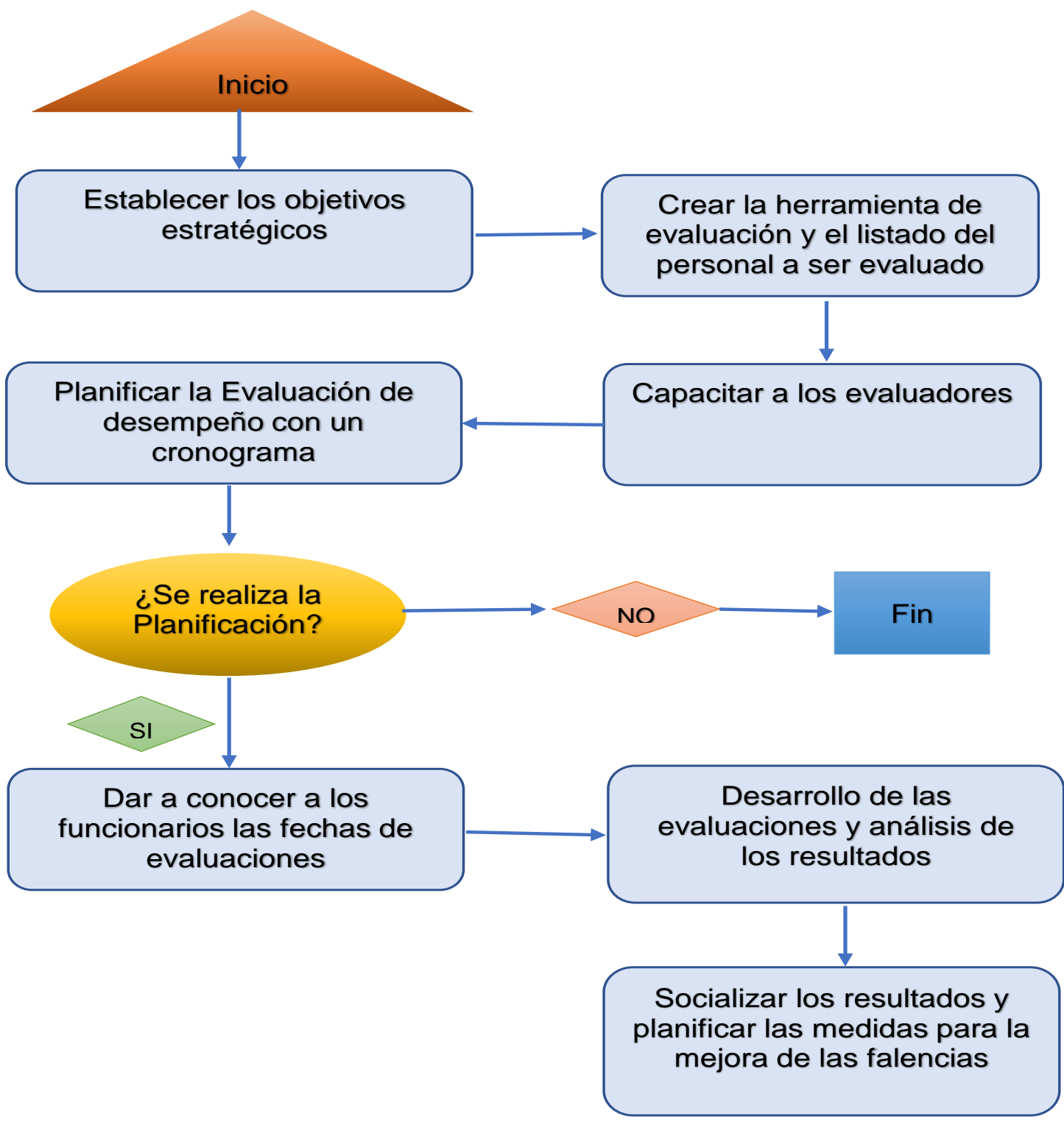

Figura 5. Flujograma del procedimiento de evaluación.

Elaboración: Los autores. 


\section{Evaluación de $360^{\circ}$ usuarios internos y externos}

En esta etapa final, y de acuerdo a los resultados obtenidos en el proceso de evaluación se propone en la figura 6 , el plan de mejora continua, en la misma se ha considerado varias fases que permitan realizar el seguimiento y monitoreo, de esta manera tener la certeza de que se está cumpliendo con la misma.

\begin{tabular}{|c|c|c|c|c|c|c|c|c|c|}
\hline $\begin{array}{c}\text { Aspecto a } \\
\text { mejorar }\end{array}$ & $\begin{array}{c}\text { Plan de } \\
\text { acción }\end{array}$ & $\begin{array}{c}\text { Actividades } \\
\text { del Plan de } \\
\text { acción }\end{array}$ & $\begin{array}{c}\text { Indicador } \\
\text { del logro }\end{array}$ & Meta & $\begin{array}{c}\text { Medios de } \\
\text { verificación }\end{array}$ & Recursos & Responsable & $\begin{array}{c}\text { Fecha de } \\
\text { inicio }\end{array}$ & $\begin{array}{c}\text { Fecha de } \\
\text { termino }\end{array}$ \\
\hline $\begin{array}{c}\text { Corresponde a } \\
\text { las debilidades } \\
\text { identificadas en } \\
\text { el proceso de } \\
\text { autoevaluación }\end{array}$ & $\begin{array}{c}\text { Co un plan } \\
\text { específico } \\
\text { que apunta a } \\
\text { mejorar una } \\
\text { debilidad }\end{array}$ & $\begin{array}{c}\text { Tareas o } \\
\text { eventos } \\
\text { destinados al } \\
\text { cumplimiento } \\
\text { del plan de } \\
\text { acción }\end{array}$ & $\begin{array}{c}\text { Parámetros } \\
\text { de medida } \\
\text { por medio } \\
\text { de los } \\
\text { cuales se } \\
\text { determina el } \\
\text { logro de la } \\
\text { meta }\end{array}$ & $\begin{array}{c}\text { Corresponde } \\
\text { al } \\
\text { compromiso la actividad } \\
\text { de } \\
\text { cumplimiento }\end{array}$ & $\begin{array}{c}\text { Evidencia } \\
\text { que permite } \\
\text { comprobar } \\
\text { que la } \\
\text { actividad del } \\
\text { plan de } \\
\text { acción se } \\
\text { llevó a cabo }\end{array}$ & $\begin{array}{c}\text { Recursos } \\
\text { (monetarios, } \\
\text { horas } \\
\text { hombre, } \\
\text { espacios } \\
\text { físicos, etc. }) \\
\text { necesarios } \\
\text { para el logro } \\
\text { de una } \\
\text { actividad }\end{array}$ & $\begin{array}{c}\text { Persona que para que } \\
\text { se cumpla la } \\
\text { actividad }\end{array}$ & $\begin{array}{c}\text { Fecha en } \\
\text { la cual se } \\
\text { debe } \\
\text { comenzar } \\
\text { la actividad }\end{array}$ & $\begin{array}{c}\text { Fecha en } \\
\text { la cual se } \\
\text { debe } \\
\text { finalizar la } \\
\text { actividad }\end{array}$ \\
\hline
\end{tabular}

Figura 6. Matriz de Implementación del plan de mejora continua.

\section{CONCLUSIONES}

El Hospital Homero Castanier Crespo debe tomar en consideración la propuesta de capacitar a los servidores públicos que cumplen cargos administrativos en las diferentes áreas, esto permitirá que los funcionarios se formen profesionalmente y adquieran nuevos conocimientos que contribuyan a mejorar su desempeño, y a su vez servirá de motivación para alcanzar los objetivos Institucionales.

Del análisis realizado al resultado de las encuestas, se determina que en el Hospital Homero Castanier Crespo existe la inconformidad de los funcionarios ya que no están ocupando los cargos para los que fueron contratados, por tal razón es imprescindible que 
Revista Arbitrada Interdisciplinaria KOINONIA

Año VI. Vol VI. N 12. Julio - Diciembre. 2021

Hecho el depósito de Ley: FA2016000010

ISSN: $2542-3088$

FUNDACIÓN KOINONIA (F.K). Santa Ana de Coro. Venezuela.

María Angélica Heredia-Quito; Cecilia Ivonne Narváez-Zurita

la Unidad de Talento Humano realice la reclasificación del personal acorde a sus competencias y perfiles.

Así también, la Institución debe aplicar de manera correcta el Manual de Descripción, valoración y clasificación de puestos de Hospitales, para evitar la insatisfacción del personal al verse afectados de manera económica y profesional.

Esta Casa de Salud al no poseer una herramienta integral de evaluación de desempeño, se propone aplicar la evaluación de desempeño $360^{\circ}$, de esta manera se podrá optimar el desempeño de los funcionarios del Hospital y mejorar la gestión administrativa.

De igual manera se propone que exista un correcto proceso de planificación participativa de la evaluación de desempeño, seguido de un plan de mejora, siendo la Unidad de Talento Humano los responsables de la ejecución, control y seguimiento.

\section{FINANCIAMIENTO}

No monetario.

\section{AGRADECIMIENTO}

A las autoridades del Hospital Homero Castanier Crespo, quienes consintieron llevar a cabo esta investigación y a la Jefatura de Posgrados de la Universidad Católica de Cuenca por el apoyo permanente.

\section{REFERENCIAS CONSULTADAS}

Alfonso, M. (2016). La evaluación del desempeño: pasado, presente y futuro [Performance evaluation: past, present and future]. Obtenido de https://n9.cl/dclr

Alles, M. (2006). Dirección Estratégica de Recursos Humanos [Strategic Human Resources Management]. Argentina: Granica. 


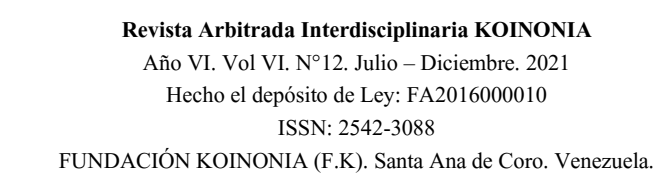

María Angélica Heredia-Quito; Cecilia Ivonne Narváez-Zurita

Amador-Licona, N, Aguirre García, M, Anguiano Peña, N, \& Guízar Mendoza, J. (2018). Desempeño laboral de acuerdo al estado de salud del trabajador y el uso del móvil en organizaciones laborales [Work performance according to the work health status and use of the mobile in laboral organizations]. Nova scientia, 10(21), 423440. https://doi.org/10.21640/ns.v10i21.1406

Argudo-Tello, K., Erazo Álvarez, J., \& Narváez Zurita, C. (2019). Evaluación de Control Interno en Riesgos Estratégicos para la Dirección de Planificación de la Universidad de Cuenca [Evaluation of Internal Control in Strategic Risks for the Planning Department of the University of Cuenca]. Revista Arbitrada Interdisciplinaria Koinonía, 4(1), 67-96. http://dx.doi.org/10.35381/r.k.v4i1.372

Bernal Ávila, E., Erazo Álvarez, J., \& Narváez Zurita, C. (2019). Estructuras organizativas favorables a la Eficiencia Empresarial [Organizational structures favorable to Business Efficiency]. Revista Arbitrada Interdisciplinaria Koinonía, 4(1), 3-31. http://dx.doi.org/10.35381/r.k.v4i1.370

Bittel. (2000). Administración de personal [Personnel administration]. Traducción Jose Real Gutierres.

Brazzolotto, S. (2012). Aplicación de la Evaluación de Desempeño por competencias a las Organizaciones [Application of Competency-Based Performance Evaluation to Organizations]. Universidad Nacional de Cuyo, Facultad de Ciencias Económicas, Mendoza. https://n9.cl/w26u

Buelvas., P., et al. (2002). Métodos de evaluación del desempeño laboral [Job performance evaluation methods]. Recuperado desde https://n9.cl/6whn8

Carrión-Agila, E., Erazo Álvarez, J., Narváez Zurita, C., \& Trelles Vicuña, D. (2019). La lógica difusa como herramienta para la evaluación del desempeño de los servidores públicos [Fuzzy logic as a tool for evaluating the performance of public servants]. CIENCIAMATRIA, 5(1), 215-244. https://doi.org/10.35381/cm.v5i1.265

Cejas, M., Vasquez, G., Chirinos, N., Hernandez, G., Sandoval, L., Lozada, B., \& Anzola, A. (2012). Administración de Recursosn Humanos. La arquitectura estratégica de las organizaiones[Human Resources Administration. The strategic architecture of organizations]. (D. A. Aguirre, Ed.) Sangolquí: David Cabrera Reinoso.

Chiavenato. (2000). Administración de los Recursos Humanos [Human Resources Management]. Bogotá: McGRAW-HILL/INTERAMERICANA EDITORES, S.A. DE C.V. 


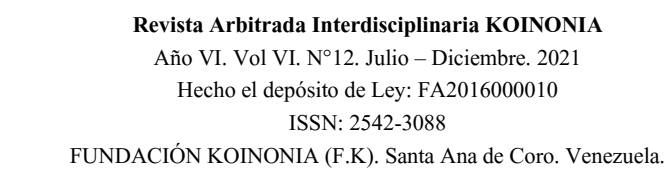

María Angélica Heredia-Quito; Cecilia Ivonne Narváez-Zurita

Cordero, J., Narváez, C., \& Erazo, J. (2019). La evaluación por competencias: una herramienta para determinar la productividad del talento humano [Assessment by competencies: a tool to determine the productivity of human talent]. 593 Digital Publisher CEIT, 4(3-1), 76-96. https://doi.org/10.33386/593dp.2019.3-1.113

Hospital Homero Castanier Crespo. (2021). Plan Estratégico Institucional [Institutional Strategic Plan]. Azogues: Hospital Homero Castanier Crespo. Recuperado desde https://n9.cl/ml5jg

Ivancevich, J. (2004). Administración de Recursos Humanos [Human resources management]. México: Edamsa.

Koontz, H., Weihrich, H., \& Cannice, M. (2012). Administración, una perspectiva global y empresarial [Management, a global and business perspective.] (14va ed.). México: Mc Graw Hill.

Ley Orgánica De Servicio Público, LOSEP. Registro Oficial Suplemento 294 de 06-oct.2010. https://n9.cl/9lztn

Münch. (2010). Administración Gestión Organizacional, enfoques y procesos administrativos[ Management Organizational Management, administrative approaches and processes]. Mexico: Pearson Educación de México, S.A. de C.V.

Ortiz-Andrade, E. J., \& Rivas-Quezada, P. (2011). Propuesta de un manual de planes de carrera en cargos administrativos [Proposal for a manual of career plans in administrative positions]. http://dspace.uazuay.edu.ec/handle/datos/1759

Pedraza, E., Amaya, G., \& Conde, M. (2010). Desempeño Laboral y estabilidad del peronal Administrativo contratado de la Facultad de Medicina de la Universidad del Zulia. Revista de Ciencias Sociales (Venezuela), Vol. XVI, Vol. XVI(3), 493-505.

Saldoval, L. (2010). Administración de los Recursos Humanos [Human Resources Administration]. Sangolquí: Editorial de la Universidad de las Fuerzas Armadas ESPE.

Sarries-Sanz, L., \& Casares-García, E. (2008). Buenas prácticas de Recursos Humanos [Good Human Resources practices]. Madrid: ESIC EDITORIAL.

SNGSP. (2012). Manual del modelo integral de atención integral de salud-MAIS [Comprehensive Health Care Model Manual-MAIS]. QUITO: MSP. https://n9.cl/xre2n 
Universidad de Champagnat. (2002). Capacitación y desarrollo profesional del personal [Staff training and professional development]. Recuperado desde https://n9.cl/z87eun

Urgilés-Urgilés, S., Erazo Álvarez, J., \& Narváez Zurita, C. (2019). El coaching y la productividad laboral en la Cooperativa de Ahorro y Crédito de la Pequeña Empresa Biblián Ltda [Coaching and labor productivity in the Small Business Savings and Credit Cooperative Biblián Ltda]. Revista Arbitrada Interdisciplinaria Koinonía, 4(1), 408-435. http://dx.doi.org/10.35381/r.k.v4i1.463

Vance Mafla, K. (2012). Estatuto, gestión, organizaciones,hospitales [Statute, management, organizations, hospitals]. Obtenido de https://n9.cl/fuoi

Wayne- Mondy, R., \& Noe, R. M. (2005). Administración de Recursos Humanos [Human Resources Management] (Novena edición ed.). Ciudad de México: Pearson Prentice Hall. 